Notre Dame Journal of Formal Logic

Volume 27, Number 1, January 1986

\title{
Notes on the Mathematical Aspects of Kripke's Theory of Truth
}

\author{
MELVIN FITTING
}

1 Introduction Kripke's Theory of Truth [3] is one of the most interesting developments to come along in this area for some time. But the mathematical difficulties may have prevented a wider appreciation of its philosophical virtues. Certainly more is required of the reader than in Tarski's approach; indeed the mathematical machinery involved strictly includes that required by Tarski's theory. But, in fact, the necessary mathematics is simple, elegant, and part of the standard tool-kit of workers in certain areas of mathematical logic and computer science.

What we do in this primarily expository paper is present, in a compact connected fashion, a development of the background mathematics pertinent to Kripke's theory. We largely skip over philosophical motivation; one cannot do better than to read Kripke himself on this. We do not present the body of the theory, only the mathematical skeleton. The paper is self-contained in the mathematical sense, however, with all terms defined and all main results proved.

There are a few items in the treatment here which, although not new, seem to have been ignored by most writers in the area.

It is most common to establish the existence of smallest fixed points by using ordinally indexed sequences of approximations. This is not necessary, and has two distinct drawbacks: First, it is more mathematical paraphernalia than one needs, tending to obscure the inherent simplicity of the subject. Second, it is a construction that only works for special kinds of fixed points, while in Kripke's theory all fixed points have some role to play. Consequently we postpone this technique to the final section; an afterthought rather than a central feature. We do show, however, that there is an analogous "dual" construction of the largest intrinsic fixed point, something that seems to have gone unremarked on in the literature.

Another peculiar feature of this work is its treatment of models. There are none. One is interested in statements, their truth and falsity. So rather than 
models, we use sets of statements directly, Hintikka fashion. Again this gets some unnecessary machinery out of the way, making, we believe, the key features of the construction stand out. And it has one satisfying side effect that in the treatment of Kleene's three-valued logic, there is no third "truth value" of undefined. We have only the usual two truth values, but not every statement acquires a truth value. A minor point, but a satisfying one.

We must point out that none of the results here are new. A few items can definitely be ascribed to particular authors, most seem to be part of the mathematical folklore. Primarily we are attempting to provide a service: collecting this material in one place, and organizing it with Kripke's work in mind.

2 Monotone operators Suppose we create an operator that takes as input a collection of assertions and produces as output the collection of "simple" consequences of these assertions. Such an operator is monotone in the sense that if we supply additional input, the output does not diminish. A special role is played by those collections of assertions that, when used as input, are returned unchanged as output. Such fixed points amount to collections of assertions that are complete, in that they already contain their consequences. The existence and variety of such fixed points play a fundamental role in Kripke's theory. And the mathematical machinery to deal with them can be presented most simply in an abstract, lattice theoretic setting.

Definitions $\langle\mathbf{D}, \leq\rangle$ is a partial ordering if $\leq$ is a relation on $\mathbf{D}$ that is reflexive, antisymmetric, and transitive. Throughout this section, we assume $\leq$ is some fixed partial ordering on D. If $A \leq B$ we say $A$ is below $B$ and $B$ is above $A$. We also use the term between in the obvious way.

Let $\mathbf{C} \subseteq \mathbf{D}$. The largest member of $\mathbf{C}$ is an $A \in \mathbf{C}$ such that $B \leq A$ for all $B \in \mathbf{C}$. If $\mathbf{C}$ has a largest member, it is easily seen to be unique. Smallest has a dual definition.

Let $\varnothing \neq \mathbf{C} \subseteq \mathbf{D}$. An upper bound for $\mathbf{C}$ is a member $A$ of $\mathbf{D}$ such that $B \leq A$ for all $B \in \mathbf{C}$. An upper bound for $\mathbf{C}$ need not be a member of $\mathbf{C}$, nor need one exist at all. Lower bounds have a dual definition. Note that if $\mathbf{C}$ has an upper bound belonging to $\mathbf{C}$, it is the largest member of $\mathbf{C}$.

Again let $\varnothing \neq \mathbf{C} \subseteq \mathbf{D}$. If $\mathbf{C}$ has upper bounds, and among them there is a smallest, it is the least upper bound of $\mathbf{C}$. If it exists it is unique, and is denoted by $\mathrm{V}$. Note that if $\mathbf{C}$ has a least upper bound then

1. $A \in \mathbf{C} \Rightarrow A \leq \bigvee \mathbf{C}$

2. $A \leq B$ for all $A \in \mathbf{C} \Rightarrow \bigvee \mathbf{C} \leq B$.

Greatest lower bounds have a dual characterization. The notation used is $\wedge \mathbf{C}$. If $\mathbf{C}$ has a greatest lower bound then

1. $A \in \mathbf{C} \Rightarrow \bigwedge \mathbf{C} \leq A$

2. $B \leq A$ for all $A \in \mathbf{C} \Rightarrow B \leq \bigwedge \mathbf{C}$.

Let $\Phi: \mathbf{D} \rightarrow$ D. $\Phi$ is monotone or order preserving if $A \leq B \Rightarrow \Phi(A) \leq$ $\Phi(B)$. If $\Phi(A)=A$ then $A$ is a fixed point of $\Phi$.

The fundamental theorem in the subject is the following, due to Knaster [2] in a set theoretic context, generalized to the present extent by Tarski [5]. 
Fixed Point Theorem 2.1 Let $\langle\mathrm{D}, \leq\rangle$ be a partial ordering and let $\Phi$ be monotone. Suppose there are $A, B \in \mathbf{D}$ such that $A \leq \Phi(A), A \leq B$ and $\Phi(B) \leq B$.

(1) If every nonempty subset of $\mathbf{D}$ has a greatest lower bound in $\mathbf{D}$ then $\Phi$ has a smallest fixed point between $A$ and $B$.

(2) If every nonempty subset of $\mathbf{D}$ has a least upper bound in $\mathbf{D}$ then $\Phi$ has a largest fixed point between $A$ and $B$.

Proof: The proof of (2) is the dual of that of (1). We only show (1).

Let $\mathbf{C}=\{X \in \mathbf{D} \mid A \leq X$ and $\Phi(X) \leq X\}$. By hypothesis, $B \in \mathbf{C}$ so $\mathbf{C}$ is not empty.

Suppose $X \in \mathbf{C}$. Then $A \leq X$ and $\Phi(X) \leq X$. By monotonicity, $\Phi(A) \leq$ $\Phi(X)$ and $\Phi(\Phi(X)) \leq \Phi(X)$. By hypothesis, $A \leq \Phi(A)$, hence $A \leq \Phi(X)$. It follows that $\Phi(X) \in \mathbf{C}$. Thus $\mathbf{C}$ is closed under $\Phi$.

By hypothesis, $\bigwedge \mathbf{C} \in \mathbf{D}$. We claim it is the desired fixed point. Note that for all $X \in \mathbf{C}, A \leq X$, hence $A \leq \wedge \mathbf{C}$. Also $B \in \mathbf{C}$, so $\bigwedge \mathbf{C} \leq B$. Thus $\bigwedge \mathbf{C}$ is between $A$ and $B$.

Suppose $X \in \mathbf{C}$. Then $\wedge \mathbf{C} \leq X$. By monotonicity, $\Phi(\wedge \mathbf{C}) \leq \Phi(X)$. But since $X \in \mathbf{C}, \Phi(X) \leq X$, so $\Phi(\wedge \mathbf{C}) \leq X$. Since $X$ was an arbitrary member of $\mathbf{C}, \Phi(\wedge \mathbf{C}) \leq \wedge \mathbf{C}$.

It follows that $\wedge \mathbf{C} \in \mathbf{C}$. Since $\mathbf{C}$ is closed under $\Phi, \Phi(\wedge \mathbf{C}) \in \mathbf{C}$. Hence $\wedge \mathbf{C} \leq \Phi(\wedge \mathbf{C})$

Thus, by antisymmetry, $\bigwedge \mathbf{C}$ is a fixed point of $\Phi$. Finally, if $F$ is any fixed point extending $A, F \in \mathbf{C}$ so $\bigwedge \mathbf{C} \leq F$. Hence $\bigwedge \mathbf{C}$ is smallest.

Remark: This theorem is also the basis for a method of proof by "generalized induction" as follows:

Suppose the hypotheses of (1) are met. Let $C \in \mathbf{D}$ and suppose we wish to show the smallest fixed point of $\Phi$ above $A$ must be below $C$. This will be the case provided

$A \leq C$

whenever $X \leq C$ then $\Phi(X) \leq C$.

Reason: let $C$ play the role of $B$ in the Fixed Point Theorem. Since $C \leq C$ the second condition implies $\Phi(C) \leq C$. Then the smallest fixed point of $\Phi$ above $A$ is guaranteed to be below $B$, that is, below $C$ in this case.

More Definitions As before $\langle\mathbf{D}, \leq\rangle$ is some fixed partial ordering.

$\mathbf{C} \subseteq \mathbf{D}$ is a chain if, for any $A, B \in \mathbf{C}, A \leq B$ or $B \leq A$.

$M \in \mathbf{D}$ is maximal if $M \leq A \Rightarrow M=A$. $M$ is minimal if $A \leq M \Rightarrow A=M$.

$A, B \in \mathbf{D}$ are compatible if they have a common upper bound, that is, for some $C \in \mathbf{D}, A \leq C$ and $B \leq C$. 
For a monotone $\Phi$ on $\mathbf{D}, I$ is an intrinsic fixed point of $\Phi$ if $I$ is a fixed point that is compatible with every fixed point of $\Phi$.

Main Theorem 2.2 Let $\langle\mathbf{D}, \leq\rangle$ be a partial ordering in which $\mathbf{D}$ has a smallest member, every chain has an upper bound, and every nonempty set having an upper bound has a least upper bound. Also let $\Phi$ be monotone on $\mathbf{D}$. Then the following consequences hold:

(C1) Every nonempty subset of $\mathbf{D}$ has a greatest lower bound in $\mathbf{D}$.

(C2) If $A \leq \Phi(A)$ then there is a maximal fixed point of $\Phi$ above $A$.

(C3) $\Phi$ has maximal fixed points.

(C4) If $A \leq \Phi(A)$ then there is a smallest fixed point of $\Phi$ above $A$.

(C5) $\Phi$ has a smallest fixed point.

(C6) If $\Phi(B) \leq B$ then there is a largest fixed point of $\Phi$ below $B$

(C7) Let I be a fixed point of $\Phi$. I is intrinsic if and only if $I \leq \wedge \mathbf{M}$ where $\mathbf{M}$ is the set of maximal fixed points of $\Phi$.

(C8) $\Phi$ has a largest intrinsic fixed point.

(C9) If $A \leq \Phi(A)$ and $A \leq \bigwedge \mathbf{M}$ where $\mathbf{M}$ is the set of maximal fixed points of $\Phi$, then the smallest fixed point of $\Phi$ above $A$ is intrinsic.

Remark: $A$ is sometimes called sound if $A \leq \Phi(A)$. Then C2, C4, and C9 are conclusions about sound members of $\mathbf{D}$.

Proof:

(C1) Let $\mathbf{G}$ be a nonempty subset of $\mathbf{D}$. We show $\bigwedge \mathbf{G}$ exists in $\mathbf{D}$.

Define $\mathbf{E}$ to be the set of lower bounds of $\mathbf{G}$. Since $\mathbf{D}$ is assumed to have a smallest member, $\mathbf{E}$ is not empty. $\langle\mathbf{E}, \leq\rangle$ is also a partially ordered set, where $\leq$ is the original ordering restricted to $\mathbf{E}$.

We show that $\mathbf{E}$ contains, with each chain, the least upper bound of that chain. Well, let $\mathbf{C}$ be a chain in $\mathbf{E}$. By the hypotheses of Theorem 2.2, $\mathbf{C}$ has an upper bound, and hence a least upper bound in $\mathbf{D}$. That is, $\bigvee \mathbf{C} \in \mathbf{D}$. Let $Y \in$ G. For each $X \in \mathbf{C}, X \leq Y$, hence $\bigvee \mathbf{C} \leq Y$. Since $Y$ was arbitrary, $\bigvee \mathbf{C} \in \mathbf{E}$.

Since $\mathbf{E}$ is a partially ordered set in which each chain has an upper bound, $\mathbf{E}$ has maximal members, by Zorn's Lemma. Let $M$ be a maximal member of E. We claim $M$ is actually the greatest member of $\mathbf{E}$.

Let $A$ be some member of $\mathbf{E}$. Let $Y_{0}$ be some member of $\mathbf{G}$ (which must exist since $\mathbf{G}$ is nonempty). Then $A \leq Y_{0}$ and $M \leq Y_{0}$ by definition of $\mathbf{E}$. Since $\{A, M\}$ has $Y_{0}$ as an upper bound, it has a smallest upper bound, $\bigvee\{A, M\}$. It is straightforward to show that $\bigvee\{A, M\} \in \mathbf{E}$. But $M \leq \bigvee\{A, M\}$, and $M$ is maximal, so $M=\bigvee\{A, M\}$. Since $A \leq \bigvee\{A, M\}, A \leq M$. Thus $M$ is the greatest member of $\mathbf{E}$.

$\mathbf{E}$ is the set of lower bounds of $\mathbf{G}$, and $\mathbf{E}$ has a greatest member, so $\mathbf{G}$ has a greatest lower bound.

(C2) Suppose $A \leq \Phi(A)$. This time let $\mathbf{E}=\{X \in \mathbf{D} \mid X \leq \Phi(X)\}$. Since $A \leq \Phi(A), A$ is in $\mathbf{E}$. As before, $\langle\mathbf{E}, \leq\rangle$ is also a partially ordered set, where $\leq$ is the original ordering restricted to $\mathbf{E}$.

We claim every chain in $\mathbf{E}$ has an upper bound in $\mathbf{E}$. Let $\mathbf{C}$ be a chain in E. Then of course $\mathbf{C}$ is a chain in $\mathbf{D}$ too, so $\bigvee \mathbf{C} \in \mathbf{D}$. Now suppose $X \in \mathbf{C}$. Then $X \leq \bigvee \mathbf{C}$ so by monotonicity, $\Phi(X) \leq \Phi(\bigvee \mathbf{C})$. Since $X \in \mathbf{C} \subseteq \mathbf{E}$, 
$X \leq \Phi(X)$. Hence $X \leq \Phi(\bigvee \mathbf{C})$, for every $X \in \mathbf{C}$. Thus $\bigvee \mathbf{C} \leq \Phi(\bigvee \mathbf{C})$, so $\bigvee \mathbf{C}$ is in $\mathbf{E}$.

Since $\mathbf{E}$ is a partially ordered set in which each chain has an upper bound, each member of $\mathbf{E}$, in particular $A$, can be extended to a maximal member of E, by Zorn's Lemma.

Let $M$ be a maximal member of $\mathbf{E}$. We claim $M$ is a maximal fixed point of $\Phi$ in $\mathbf{D}$. Since $M \in \mathbf{E}, M \leq \Phi(M)$. By monotonicity, $\Phi(M) \leq \Phi(\Phi(M))$, hence $\Phi(M) \in \mathbf{E}$. Since $M$ is maximal in $\mathbf{E}$, it follows that $M=\Phi(M)$, hence $M$ is a fixed point. Further, if $F$ is any fixed point of $\Phi$, trivially $F \in \mathbf{E}$. But $M$ is maximal in $\mathbf{E}$, so $F$ cannot properly extend $M$. Thus $M$ is a maximal fixed point. We have shown $\mathrm{C} 2$.

(C3) By assumption, $\mathbf{D}$ has a smallest member; we denote it by 0 . Since 0 is below every member of $\mathbf{D}$, of course $0 \leq \Phi(0)$. Then by $\mathrm{C} 2$, 0 can be extended to a maximal fixed point. This establishes $\mathrm{C} 3$.

(C4) Suppose $A \leq \Phi(A)$. By $\mathrm{C} 2, \Phi$ has maximal fixed points above $A$; let $B$ be one of them. Then also $A \leq B$ and $\Phi(B) \leq B$. By part (1) of the Fixed Point Theorem, $\Phi$ has a smallest fixed point above $A$. This establishes $C 4$.

(C5) Take $A$ to be the smallest member of $\mathbf{D}$ in $\mathbf{C} 4$.

(C6) Suppose $\Phi(B) \leq B$. Let $\mathbf{G}=\{X \in \mathbf{D} \mid X \leq B\}$. As usual, $\langle\mathbf{G}, \leq\rangle$ is a partially ordered set, where $\leq$ is the restriction of the $\mathbf{D}$ ordering to $\mathbf{G}$. Every nonempty subset of $\mathbf{G}$ has an upper bound, namely $B$, and so by the theorem hypothesis, has a least upper bound in $\mathbf{D}$ which, trivially, must also be in $\mathbf{G}$.

Suppose $A \in \mathbf{G}$. Then $A \leq B$ so by monotonicity, $\Phi(A) \leq \Phi(B) \leq B$. Thus $\Phi(A) \in \mathbf{G} ; \mathbf{G}$ is closed under $\Phi$.

Let 0 be the smallest member of $\mathbf{D}$. Of course $0 \in \mathbf{G}, 0 \leq \Phi(0), B \in \mathbf{G}$, $0 \leq B$, and $\Phi(B) \leq B$. Then by the Fixed Point Theorem part 2, applied in $\mathbf{G}$, $\Phi$ has a largest fixed point in $\mathbf{G}$ between 0 and $B$. This, trivially, is the largest fixed point of $\Phi$ in $\mathbf{D}$ below $B$.

(C7) Let $\mathbf{M}$ be the set of maximal fixed points.

Suppose $I$ is an intrinsic fixed point of $\Phi$. Let $M$ be any member of $\mathbf{M}$. By the theorem hypotheses, $\bigvee\{I, M\} \in \mathbf{D}$. Now $I \leq \bigvee\{I, M\}$ hence $I=\Phi(I) \leq$ $\Phi(\bigvee\{I, M\})$. Similarly $M \leq \Phi(\bigvee\{I, M\})$. It follows that $\bigvee\{I, M\} \leq$ $\Phi(\bigvee\{I, M\})$. Then $\bigvee\{I, M\}$ is in the collection $\mathbf{E}$ defined in the proof of C2. Also $M \in \mathbf{E}$, and it must be maximal in $\mathbf{E}$ for, if not, it could be extended to a maximal member of $\mathbf{E}$ which, by the argument earlier, would be a maximal fixed point for $\Phi$, and would properly extend $M$. Since $M$ is maximal in $\mathbf{E}$, and $M \leq \bigvee\{I, M\}$, it follows that $M=\bigvee\{I, M\}$. Since $I \leq \bigvee\{I, M\}$ then $I \leq M$. And since $M$ was an arbitrary member of $\mathbf{M}, I \leq \bigwedge \mathbf{M}$.

Conversely, suppose $I$ is a fixed point of $\Phi$ and $I \leq \wedge \mathbf{M}$. We show $I$ is intrinsic. Let $F$ be any fixed point. Then $F \in \mathbf{E}$, so $F$ can be extended to a maximal member $M$ of E. $M$ is a maximal fixed point of $\Phi$ by the argument earlier, so $M \in \mathbf{M}$. Then $F \leq M$ and $I \leq \bigwedge \mathbf{M} \leq M$, so $I$ and $F$ have a common upper bound, $M$. Since $F$ was arbitrary, $I$ is intrinsic.

(C8) Let $\mathbf{M}$ be the set of maximal fixed points of $\Phi$. Let $M \in \mathbf{M}$. Then $\bigwedge \mathbf{M} \leq M$, so by monotonicity, $\Phi(\bigwedge \mathbf{M}) \leq \Phi(M)=M$. Since $M$ was an 
arbitrary member of $\mathbf{M}, \Phi(\bigwedge \mathbf{M}) \leq \bigwedge \mathbf{M}$. Now by $\mathrm{C} 6, \Phi$ has a largest fixed point below $\bigwedge \mathbf{M}$, which is the largest intrinsic fixed point by $\mathrm{C} 7$.

(C9) Finally, suppose $A \leq \Phi(A)$ and $A \leq \wedge \mathbf{M}$. As we showed in the proof of C8, $\Phi(\bigwedge \mathbf{M}) \leq \bigwedge \mathbf{M}$. Then by the Fixed Point Theorem 2.1(1), the smallest fixed point of $\Phi$ extending $A$ will be below $\bigwedge \mathbf{M}$, hence will be intrinsic by $\mathrm{C} 7$.

3 Saturated sets Kripke's aim is the creation of a theory that contains its own truth predicate. Tarski's construction, under the assumption of the existence of such a truth predicate, of a paradoxical statement asserting its own falsehood, makes the simple attainment of this goal impossible. Kripke's "out" is to allow some statements (the paradoxical one just referred to, for instance) to lack truth values. That is, there can be truth-value gaps. The question is, what rules must the assignment of truth-values to statements follow. Kripke considered several alternatives. We take up two in this section: Kleene's (strong) three-valued logic [1], and van Frassen's supervaluations [6].

Rather than work with a valuation, a function from statements to truth values, we find it more convenient and more elegant to follow Hintikka's tradition and work with a set of statements instead. Intuitively the set consists of those statements a valuation would make true, if we had valuations. And we adopt Smullyan's device [4] of signed statements: prefixing a statement with $T$ or $F$, which in fact is suggested by Kripke himself in footnote 24 of [3]. So, if $S$ is a set of signed statements, it determines a mapping to truth values as follows: For a statement $X$, if $T X \in S, X$ maps to true; if $F X \in S, X$ maps to false; if neither $T X$ nor $F X$ is in $S, X$ lacks a truth value. Notice that, in this approach, there is no special "undefined" truth value; lacking a truth value simply means that no truth value has been assigned. Now the issue is: what closure conditions do we want to impose on a set $S$ of signed statements to reflect our understanding of language and truth? Technical details follow.

For the rest of this section, $L$ is a fixed first-order language with an infinite set of constant symbols. For simplicity we assume formulas are built up using $\wedge, \sim$, and $\forall$. We use statement to mean a formula of $L$ with no free variables. A signed statement is an expression of one of the forms $T X$ or $F X$, where $X$ is a statement, and $T$ and $F$ are two fixed additional symbols.

Definitions Let $S$ be a set of signed statements.

(1) $S$ is downward saturated if
a. $T X \wedge Y \in S \Rightarrow T X \in S$ and $T Y \in S$
b. $F X \wedge Y \in S \Rightarrow F X \in S$ or $F Y \in S$
c. $T \sim X \in S \Rightarrow F X \in S$
d. $F \sim X \in S \Rightarrow T X \in S$
e. $T(\forall x) A(x) \in S \Rightarrow T A(c) \in S$ for every constant $c$
f. $F(\forall x) A(x) \in S \Rightarrow F A(c) \in S$ for some constant $c$.

(2) $S$ is upward saturated if
a. $T X \in S$ and $T Y \in S \Rightarrow T X \wedge Y \in S$
b. $F X \in S$ or $F Y \in S \Rightarrow F X \wedge Y \in S$ 
c. $F X \in S \Rightarrow T \sim X \in S$

d. $T X \in S \Rightarrow F \sim X \in S$

e. $T A(c) \in S$ for every constant $c \Rightarrow T(\forall x) A(x) \in S$

f. $F A(c) \in S$ for some constant $c \Rightarrow F(\forall x) A(x) \in S$.

(3) $S$ is saturated if $S$ is both downward and upward saturated.

(4) $S$ is consistent if not both $T X, F X$ are in $S$, for any statement $X$.

(5) $S$ is atomically consistent if not both $T A, F A$ are in $S$ for any atomic statement $A$.

(6) $S$ is complete if either $T X$ or $F X$ is in $S$ for every statement $X$.

(7) $S$ is atomically complete if either $T A$ or $F A$ is in $S$ for every atomic statement $A$.

(8) $S$ is a model set if $S$ is saturated, consistent and complete.

Note: These definitions are somewhat different than those generally in the literature. In our use, the set of all signed statements is saturated, though not consistent.

Let $\mathbf{D}$ be the collection of all sets of signed statements. $\subseteq$ is the usual subset relation. $\langle\mathbf{D}, \subseteq\rangle$ is a partial ordering closed under greatest lower bounds $(\cap)$ and least upper bounds $(U)$. follows:

Let $A$ be a set of signed statements. We define a map $\Phi_{A}: \mathbf{D} \rightarrow \mathbf{D}$ as

$$
\begin{aligned}
\Phi_{A}(S)= & A \cup \\
& \{T X \wedge Y \mid T X \in S \text { and } T Y \in S\} \cup \\
& \{F X \wedge Y \mid F X \in S \text { or } F Y \in S\} \cup \\
& \{T \sim X \mid F X \in S\} \cup \\
& \{F \sim X \mid T X \in S\} \cup \\
& \{T(\forall x) X(x) \mid T X(c) \in S \text { for every } c\} \cup \\
& \{F(\forall x) X(x) \mid F X(c) \in S \text { for some } c\} .
\end{aligned}
$$

$\Phi_{A}$ is monotone on D. The fixed points of $\Phi_{A}$ are exactly the upward saturated sets extending $A$. If $M$ is the set of all signed statements, trivially $A \subseteq M$ and $\Phi_{A}(M) \subseteq M$. Also $A \subseteq \Phi_{A}(A)$. Then by the Fixed Point Theorem 2.1(1), $\Phi_{A}$ has a smallest fixed point extending $A$.

Definition We have shown that, for a set $A$ of signed statements, there is a smallest upward saturated set extending $A$. We call it the upward saturated closure of $A$, and denote it by $A^{U}$.

Proposition 3.1 Let $A$ and $B$ be sets of signed statements. $A \subseteq B \Rightarrow$ $A^{U} \subseteq B^{U}$.

Proof: Obviously $A \subseteq B \Rightarrow \Phi_{A}(S) \subseteq \Phi_{B}(S)$ for all $S$. Now, suppose $A \subseteq B$. Trivially $\varnothing \subseteq B$. And $S \subseteq B^{U} \Rightarrow \Phi_{A}(S) \subseteq \Phi_{A}\left(B^{U}\right) \subseteq \Phi_{B}\left(B^{U}\right)=B^{U}$. Then by the Remark following the Fixed Point Theorem 2.1, the smallest fixed point of $\Phi_{A}$ is below $B^{U}$, i.e., $A^{U} \subseteq B^{U}$.

Proposition 3.2 If $A$ is downward saturated, so is $A^{U}$.

Proof: Suppose otherwise. Say $A$ is downward saturated, $T X \wedge Y \in A^{U}$, but $T X \notin A^{U}$ (the other possibilities are similar). Let $B$ be $A^{U}$ with $T X \wedge Y$ 
removed. $T X \wedge Y$ could not have been in $A$ since $A$ was downward saturated and $A \subseteq A^{U}$. Hence $A \subseteq B$. Also, $B$ is still upward saturated. But $B$ is a proper subset of $A^{U}$, which is the smallest upward saturated set extending $A$. This is impossible, so $A^{U}$ must have been downward saturated.

Corollary 3.3 If $A$ is downward saturated, $A^{U}$ is saturated.

Proposition 3.4 If $A$ is downward saturated and atomically consistent then $A$ is consistent.

Proof: It is straightforward to show that if $A$ is downward saturated and $T X, F X \in A$, where $A$ is not atomic, then $T Y, F Y \in A$ for some subformula $Y$ of $X$.

Corollary 3.5 If $A$ is downward saturated and atomically consistent then $A^{U}$ is consistent.

Proof: If $A$ is downward saturated, so is $A^{U}$. Say $A$ is also atomically consistent. $A$ and $A^{U}$ contain the same signed atomic statements, hence $A^{U}$ is atomically consistent, and thus consistent.

Proposition 3.6 If $A$ is atomically complete then $A^{U}$ is complete.

Proof: If $T X \notin A^{U}$ and $F X \notin A^{U}$ where $X$ is not atomic, then it is easy to show that, for some subformula $Y$ of $X, T Y \notin A^{U}$ and $F Y \notin A^{U}$.

Corollary 3.7 If $A$ is downward saturated, atomically consistent and atomically complete then $A^{U}$ is a model set extending $A$.

Corollary 3.8 If $A$ is downward saturated and atomically consistent. then $A$ can be extended to a model set.

Proof: Enlarge $A$ to an atomically complete set $B$ by adding exactly one of $T X$ or $F X$ for each atomic $X$ not already present. $B$ is still downward saturated, atomically consistent, and is also atomically complete. Then $B^{U}$ is a model set. But also $A \subseteq B$, hence, by Proposition 3.1, $A \subseteq A^{U} \subseteq B^{U}$.

Remarks: This corollary, essentially due to Hintikka, is the basis of most tableau completeness proofs. One attempts to find a tableau proof by systematically doing everything possible. If no proof is found, the process generates a downward saturated, atomically consistent set, from which a suitable model can be produced using Corollary 3.8. Smullyan [4] presents this style of proof in an elegant fashion.

Nonetheless, model sets, since they assign truth values to all statements, will not suit our purposes. We want the weaker notion of consistent, saturated set. Let $P(x)$ and $Q(x)$ be two predicate symbols of $L$. Consider the set $A=\{T P(a), T P(b), \ldots\}$, where $a, b, \ldots$ are all the constants of the language $L$. This set is atomically consistent and trivially downward saturated. Then $A^{U}$ is a saturated, consistent extension. It is easy to check that the following are in $A^{U}$ :

$$
\begin{aligned}
& T(\forall x) P(x) \\
& F \sim(\forall x) P(x) \\
& F \sim(\forall x) P(x) \wedge(\forall x) Q(x) .
\end{aligned}
$$


On the other hand, it is also easy to check that neither $T(\forall x) Q(x)$ nor $F(\forall x) Q(x)$ is in $A^{U}$; it is definitely not a model set.

One possible objection to using saturated, consistent sets is the following. Consider the set $A$ defined above. Since $(\forall x) Q(x)$ receives no truth value in $A^{U},(\forall x) Q(x) \vee \sim(\forall x) Q(x)$ also receives no truth value. But while $(\forall x) Q(x)$ may plausibly be something lacking a truth value, there are more than sentimental reasons for taking $(\forall x) Q(x) \vee \sim(\forall x) Q(x)$ to be true. The following presents an alternative closure notion that accomplishes precisely this. An equivalent to the characterization of $A^{U}$ we gave is: it is the intersection of the family of upward saturated sets that extend $A$. This version parallels the next construction quite closely.

Definition Let $A$ be downward saturated and atomically consistent. By $A^{V}$ we mean the intersection of the family of all model sets that extend $A$. (Note that by Corollary 3.8 this family is nonempty.)

Proposition 3.9 Suppose $A$ is downward saturated and atomically consistent. Then:

(1) $A^{V}$ is upward saturated and consistent

(2) $A^{U} \subseteq A^{V}$

(3) if $B$ is also downward saturated and atomically consistent then $A \subseteq B \Rightarrow$ $A^{V} \subseteq B^{V}$.

Proof:

(1) Every model set is upward saturated, and it is easy to check that the intersection of upward saturated sets is upward saturated. Similarly for consistency.

(2) $A^{U}$ is the smallest upward saturated set extending $A$, and hence is a subset of the upward saturated set $A^{V}$.

(3) Let $\mathbf{F}_{A}$ be the family of model sets extending $A$, and similarly for $\mathbf{F}_{B}$. Suppose $A \subseteq B$. Then any model set extending $B$ also extends $A$, so $\mathbf{F}_{B} \subseteq \mathbf{F}_{A}$. It follows that $\cap \mathrm{F}_{A} \subseteq \cap \mathbf{F}_{B}$, that is, $A^{V} \subseteq B^{V}$.

Once again, consider the set $A=\{\operatorname{TP}(a), T P(b), \ldots\}$. Since model sets are complete, $(\forall x) Q(x)$ has a truth value in each model set, and it follows that $T[(\forall x) Q(x) \vee \sim(\forall x) Q(x)]$ must be in every model set. Thus $(\forall x) Q(x) \vee$ $\sim(\forall x) Q(x)$ acquires the value true in $A^{V}$. On the other hand, one can consistently add either $T(\forall x) Q(x)$ or $F(\forall x) Q(x)$ to $A$, and extend the result to a model set using Corollary 3.8. Since $(\forall x) Q(x)$ gets different truth values in different model sets extending $A$, it has no truth value in $A^{V}$. Incidentally, since $T[(\forall x) Q(x) \vee \sim(\forall x) Q(x)]$ is in $A^{V}$ but neither $T(\forall x) Q(x)$ nor $F(\forall x) Q(x)$ is present, $A^{V}$ cannot be downward saturated.

Suppose we define a partial truth valuation $v$ as follows:

$$
v(X)=\left\{\begin{array}{l}
T \text { if } T X \in A^{U} \\
F \text { if } F X \in A^{U} \\
\text { undefined otherwise } .
\end{array}\right.
$$

$v$ meets the conditions of Kleene's three-valued logic [1].

Just as we did with $A^{U}$, we may use $A^{V}$ to define a partial truth valuation. This time we get one meeting the conditions of van Frassen's supervaluations 
[6]. We continue to work with sets of signed statements, though, rather than with partial valuations.

4 The Kripke construction We need a first-order language capable of supporting the machinery to code its own syntax. The details are not critical. For definiteness we use a language of arithmetic.

For this section let $L$ be the first-order language with $0,1,2, \ldots$ as constant symbols,,$+ *$ as function symbols, and $=$ and $\mathbf{T}$ as relation symbols $(\mathbf{T}$ is 1-place). $\mathbf{T}$ is intended to play the role of a truth predicate.

If $X$ is a statement of $L$ that does not contain $\mathbf{T}$, we say $X$ is true or false if $X$ is true or false in the standard model for arithmetic, under the obvious interpretation. The problem is to assign truth values to statements containing $\mathbf{T}$.

If $X$ is a statement of $\left.L,{ }^{\ulcorner} X\right\urcorner$ is the Gödel number of $X$, under some standard Gödel numbering.

Kripke essentially works with extension-antiextension pairs $\left\langle S_{1}, S_{2}\right\rangle$ where formulas in $S_{1}$ are "true", formulas in $S_{2}$ are "false", and formulas left out have no truth value. As Section 3 suggests, we prefer working with the set of signed formulas $\left\{T X \mid X \in S_{1}\right\} \cup\left\{F X \mid X \in S_{2}\right\}$. This is, of course, a simple variant, but it does allow connections with other parts of formal logic.

Let $\mathbf{A}$ be the set of "atomic arithmetic truths". That is, if $X$ is an atomic statement of $L$ not containing $\mathbf{T}$, then if $X$ is true, $T X \in \mathbf{A}$, and if $X$ is false, $F X \in \mathbf{A}$.

Note that both $A^{U}$ and $A^{V}$ are completely determined by the signed atomic statements they contain. Thus we can confine members of $\mathbf{D}$ in the following to the atomic level, which allows us to use the same $\mathbf{D}$ for both $U$ and $V$ completions.

Let $\mathbf{D}$ consist of all sets $S$ of signed atomic statements of $L$ (including those involving $\mathbf{T})$ such that:

1. $\mathbf{A} \subseteq S$

2. $S$ is (atomically) consistent.

$\langle\mathbf{D}, \subseteq\rangle$ is a partial ordering, where $\subseteq$ is the usual subset relation. $\mathbf{D}$ is closed under $\cap$ but not under $U$, since the union of consistent sets need not be consistent. However, it is straightforward to check that $\langle\mathbf{D}, \subseteq\rangle$ satisfies the conditions of the Main Theorem 2.2. A itself is the smallest member.

Next we define two operators $\Phi_{U}$ and $\Phi_{V}$ on $\mathbf{D}$, corresponding to the two notions of closure defined in the previous section. We take them one at a time.

For $S \in \mathbf{D}$

$$
\begin{aligned}
\Phi_{U}(S)= & \mathbf{A} \cup \\
& \left\{T \mathbf{T}(\ulcorner X\urcorner) \mid T X \in S^{U}\right\} \cup \\
& \left\{F \mathbf{T}(\ulcorner X\urcorner) \mid F X \in S^{U}\right\} .
\end{aligned}
$$

Let $S \in \mathbf{D}$. $S$ consists of signed atomic statements, hence is trivially downward saturated, and atomically consistent by definition. Then $S^{U}$ is saturated and consistent. Since $\mathbf{A} \subseteq S$ it follows easily that all true signed statements of arithmetic are in $S^{U}$. Since $S^{U}$ is consistent, $\left\{T \mathbf{T}(\ulcorner X\urcorner) \mid T X \in S^{U}\right\} \cup$ $\left\{F \mathbf{T}(\ulcorner X\urcorner) \mid F X \in S^{U}\right\}$ is atomically consistent. Certainly $\mathbf{A}$ is atomically consistent. Since the members of $\mathbf{A}$ do not contain $\mathbf{T}$, these sets do not involve 
common statements, so the union, $\Phi_{U}(S)$, is atomically consistent. $\Phi_{U}(S)$ extends $\mathbf{A}$ by definition. Then $\Phi_{U}(S)$ is a member of $\mathbf{D}$.

We showed in Proposition 3.1 that $A \subseteq B \Rightarrow A^{U} \subseteq B^{U}$. It follows that $\Phi_{U}$ is monotone. Thus we have a monotone operator $\Phi_{U}: \mathbf{D} \rightarrow \mathbf{D}$.

Remark: Recall, a set $S \in \mathbf{D}$ is sound if $S \subseteq \Phi_{U}(S)$. Soundness essentially says $S$ does not call anything true that it shouldn't. For example, if $T \mathbf{T}(\ulcorner X\urcorner)$ is in $S$ but $T X$ is not in $S^{U}, S$ will not be sound since $T \mathbf{T}(\ulcorner X\urcorner)$ will not be in $\Phi_{U}(S)$.

Now the definition of $\Phi_{V}$ should be obvious. For $S \in \mathbf{D}$,

$$
\begin{aligned}
\Phi_{V}(S)= & \mathbf{A} \cup \\
& \left\{T \mathbf{T}(\ulcorner X\urcorner) \mid T X \in S^{V}\right\} \cup \\
& \left\{F \mathbf{T}(\ulcorner X\urcorner) \mid F X \in S^{V}\right\} .
\end{aligned}
$$

The same argument as above shows that $\Phi_{V}: \mathbf{D} \rightarrow \mathbf{D}$. And Proposition 3.9(3) implies that $\Phi_{V}$ is monotone.

By the Main Theorem 2.2, both $\Phi_{U}$ and $\Phi_{V}$ have fixed points. If $S$ is a fixed point of $\Phi_{U}$ then $S^{U}$ essentially contains its own truth predicate. That is, $S^{U}$ makes $X$ true $\left(T X \in S^{U}\right)$ if and only if $S^{U}$ makes $\mathbf{T}(\ulcorner X\urcorner)$ true. Similarly for $S^{V}$ where $S$ is a fixed point of $\Phi_{V}$. Of course, some statements will be left without truth values. Reasonable people can (and do) differ on whether the $U$-version, or the $V$-version, or some other version altogether, gives truth values to the most statements in a way that is compatible with our understanding of the logical connectives and quantifiers.

Of course, both $\Phi_{U}$ and $\Phi_{V}$ have many fixed points. Not all are of equal importance, however. We state the following for $\Phi_{U}$, but of course analogous things apply to $\Phi_{V}$.

The smallest fixed point of $\Phi_{U}$ (guaranteed to exist by Theorem 2.2(C5) is most fundamental. Kripke calls a statement grounded if it has a truth value in $S^{U}$, where $S$ is the smallest fixed point of $\Phi_{U}$. Neither a statement asserting its own truth nor a statement asserting its own falsehood is grounded.

Kripke calls a statement paradoxical if it has no truth value in $S^{U}$ for any fixed point $S$ of $\Phi_{U}$. A statement asserting its own falsehood is paradoxical; a statement asserting its own truth is not. Since every fixed point can be extended to a maximal fixed point (by Theorem 2.2(C2)), one can equally well say a paradoxical statement is one that has no truth value in $M^{U}$ for any maximal fixed point $M$ of $\Phi_{U}$. Thus the maximal fixed points play a significant role, but there is no reason to single one out as being more fundamental than the rest.

There is no largest fixed point. There is, however, a largest intrinsic fixed point (by Theorem 2.2(C8)). Kripke calls a statement intrinsic if it has a truth value in $I^{U}$ for some intrinsic fixed point $I$ of $\Phi_{U}$. This is equivalent to saying it has a truth value in $B^{U}$ where $B$ is the largest intrinsic fixed point of $\Phi_{U}$.

One might wonder about the relationships that entail between the fixed points of $\Phi_{U}$ and $\Phi_{V}$. There are a few easy results to be had here.

First we note that, for each $S, \Phi_{U}(S) \subseteq \Phi_{V}(S)$. This follows immediately from Proposition 3.9 and the definitions of $\Phi_{U}$ and $\Phi_{V}$. 
Proposition 4.1 The smallest fixed point of $\Phi_{U}$ is below the smallest fixed point of $\Phi_{V}$.

Proof: We use the generalized induction method outlined in the Remark following the Proof of Theorem 2.1.

Let $S$ be the smallest fixed point of $\Phi_{V}$.

Trivially $\mathbf{A} \subseteq S$.

Suppose $A \subseteq S$. By monotonicity, $\Phi_{U}(A) \subseteq \Phi_{U}(S)$. By the observation above, $\Phi_{U}(S) \subseteq \Phi_{V}(S)=S$. Then $\Phi_{U}(A) \subseteq S$.

It follows that the smallest fixed point of $\Phi_{U}$ (extending $\mathbf{A}$ ) is below $S$.

Proposition 4.2 Every fixed point of $\Phi_{U}$ has a smallest fixed point of $\Phi_{V}$ above it.

Proof: Let $S$ be a fixed point of $\Phi_{U}$. Then $S=\Phi_{U}(S) \subseteq \Phi_{V}(S)$. Now use Theorem 2.2(C2).

Proposition 4.3 Every fixed point of $\Phi_{V}$ has a largest fixed point of $\Phi_{U}$ below it.

Proof: Let $S$ be a fixed point of $\Phi_{V}$. Let $\langle\mathbf{D}, \subseteq\rangle$ be the partial ordering defined earlier in this section. Let $\mathbf{E}=\{A \in \mathbf{D} \mid A \subseteq S\}$. Then $\langle\mathbf{E}, \subseteq\rangle$ is also a partial ordering. But $\mathbf{E}$ has a largest member, $S$. Trivially, then, every nonempty subset of $\mathbf{E}$ has an upper bound, namely $S$, and it follows that it has a least upper bound.

Suppose $A \in \mathbf{E}$. Then $A \subseteq S$, so $\Phi_{U}(A) \subseteq \Phi_{U}(S) \subseteq \Phi_{V}(S)=S$. Thus $\mathbf{E}$ is closed under $\Phi_{U}$.

$S \in \mathbf{E}$, hence $\Phi_{U}(S) \in \mathbf{E}$, and so $\Phi_{U}(S) \subseteq S$. Now by the Fixed Point Theorem 2.1(2), $\Phi_{U}$ has a largest fixed point below $S$.

Remark: By this proposition, any maximal fixed point of $\Phi_{V}$ has a largest fixed point of $\Phi_{U}$ below it. Suppose it could be shown that any maximal fixed point of $\Phi_{V}$ has a maximal fixed point of $\Phi_{U}$ below it. Then it could also be shown that the largest intrinsic fixed point of $\Phi_{U}$ is a subset of the largest intrinsic fixed point of $\Phi_{V}$.

5 Approximating fixed points In Kripke's paper, and in many papers based on it, the existence of a smallest fixed point of a monotone operator is established by using a transfinite sequence of approximations from below. We have chosen not to base our development on this method. Nevertheless, it does provide one with a good feeling for the character of the smallest fixed point. It is a limited technique in that there may be fixed points not obtainable by this method. It does, however, apply to the largest intrinsic fixed point, in a "dualized" form. In this section we sketch the method, partly to make contact with other papers, partly because many people seem unfamiliar with the dual version.

For this section we suppose $\langle\mathbf{D}, \leq\rangle$ is a partial ordering satisfying the hypotheses of the Main Theorem 2.2, and we assume $\Phi$ is monotone.

Let $A$ be a sound member of $\mathbf{D}$, that is, $A \leq \Phi(A)$. We define a transfinite sequence of members of $\mathbf{D}$, indexed by ordinals, as follows. 
$A_{0}=A$

$A_{\alpha+1}=\Phi\left(A_{\alpha}\right)$

$A_{\lambda}=\bigvee\left\{A_{\alpha} \mid \alpha<\lambda\right\}$ for limit ordinals $\lambda$.

This is an increasing sequence in the sense that $A_{\alpha} \leq A_{\alpha+1}$ for all $\alpha$. The proof is by (transfinite) induction on $\alpha$.

Case 0: $A_{0} \leq A_{1}$ says $A \leq \Phi(A)$ which is true since $A$ is sound.

Successor case: Suppose $A_{\alpha} \leq A_{\alpha+1}$. Then by monotonicity, $\Phi\left(A_{\alpha}\right) \leq \Phi\left(A_{\alpha+1}\right)$ which says that $A_{\alpha+1} \leq A_{\alpha+2}$.

Limit case: Suppose $\lambda$ is a limit ordinal and, for each $\alpha<\lambda, A_{\alpha} \leq A_{\alpha+1}$. Choose an $\alpha<\lambda$. By definition of $A_{\lambda}, A_{\alpha} \leq A_{\lambda}$. Hence $\Phi\left(A_{\alpha}\right) \leq \Phi\left(A_{\lambda}\right)$ or $A_{\alpha+1} \leq A_{\lambda+1}$. Using the induction hypothesis, it follows that $A_{\alpha} \leq A_{\lambda+1}$. Since $\alpha$ was arbitrary, $\bigvee\left\{A_{\alpha} \mid \alpha<\lambda\right\} \leq A_{\lambda+1}$, or $A_{\lambda} \leq A_{\lambda+1}$.

Remark: Another way of stating this is that every member of the sequence is sound.

Thus the $A_{\alpha}$ sequence is weakly increasing. Suppose we write $<$ to mean $\leq$ but not $=$. We certainly cannot have that the sequence is strongly increasing $\left(A_{\alpha}<A_{\alpha+1}\right)$, because it would follow that all members of the sequence are distinct. But then there would be as many as there are ordinals, while all are members of the set $\mathbf{D}$, a clear impossibility. Then, for some $\alpha$, we must have $A_{\alpha}=\Phi\left(A_{\alpha}\right)$; some member of the sequence is a fixed point.

Incidentally, once a fixed point turns up in the sequence, the sequence remains constant from that point on.

Suppose $F$ is a fixed point of $\Phi$ extending $A$. Then $A_{0} \leq F$ of course.

Say $A_{\alpha} \leq F$. Then $A_{\alpha+1}=\Phi\left(A_{\alpha}\right) \leq \Phi(F)=F$, so $A_{\alpha+1} \leq F$.

Say $A_{\alpha} \leq F$ for every $\alpha<\lambda$. It follows immediately that $A_{\lambda} \leq F$.

Then by induction again, every member of the sequence is $\leq F$. Since $F$ was arbitrary, it follows that the fixed point the sequence eventually hits on is the smallest fixed point extending $A$.

If we start with the smallest member of $\mathbf{D}$, we will have a sequence of approximations, from below, to the smallest fixed point of $\Phi$.

Next we sketch a dualized version of this. Choose a member $B \in \mathbf{D}$ such that $\Phi(B) \leq B$. This time define a sequence as follows.

$B_{0}=B$

$B_{\alpha+1}=\Phi\left(B_{\alpha}\right)$

$B_{\lambda}=\bigwedge\left\{B_{\alpha} \mid \alpha<\lambda\right\} \lambda$ a limit ordinal.

This time it can be shown that the sequence is decreasing, $B_{\alpha+1} \leq B_{\alpha}$, and that it eventually settles on the largest fixed point of $\Phi$ below $B$. We leave the argument to the reader.

If we start with $\bigwedge \mathbf{M}$, where $\mathbf{M}$ is the set of maximal fixed points of $\Phi$, the sequence converges to the largest intrinsic fixed point.

One sometimes thinks of the terms in the $A_{\alpha}$ sequence earlier as stages in a "learning" process that goes on transfinitely long, though not forever. Then the terms in the $B_{\alpha}$ sequence are probably best thought of as stages in 
a "forgetting" process. The smallest fixed point, being below the largest intrinsic fixed point, it follows that in these processes one cannot forget more than one learns. This seems like a comforting point at which to conclude.

\section{REFERENCES}

[1] Kleene, S. C., Introduction to Matamathematics, Van Nostrand, New York, 1952.

[2] Knaster, B., "Un théorème sur les fonctions d'ensembles," Annals de la Societé Polonaise de Mathematiques, vol. 6 (1928), pp. 133-134.

[3] Kripke, S., "Outline of a theory of truth," The Journal of Philosophy, vol. 72 (1975), pp. 690-716.

[4] Smullyan, R., First-Order Logic, Springer-Verlag, Berlin, 1968.

[5] Tarski, A., "A lattice-theoretical fixpoint theorem and its applications," Pacific Journal of Mathematics, vol. 5 (1955), pp. 285-309.

[6] van Frassen, B., "Singular terms, truth-value gaps, and free logic," The Journal of Philosophy, vol. 63 (1966), pp. 481-485.

Department of Mathematics and Computer Science

Herbert H. Lehman College

Bedford Park Boulevard West

Bronx, New York 10468 